
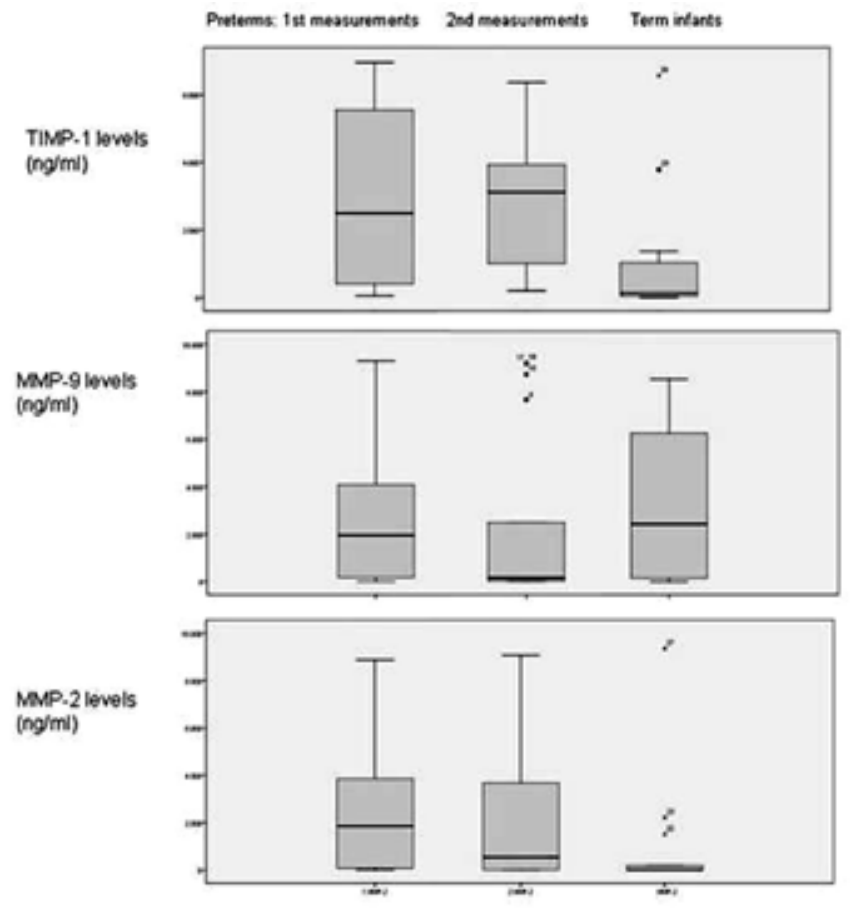

Abstract 1711 Figure 1 Comparison of MMP-2, MMP-9, and TIMP-1 levels

In contrast, there was no significant relationship between MMP-9 levels or the MMP-9/TIMP-1 ratio between preterm and term infants (univariate analysis). The area under the ROC curve for MMP-2 was 0.70 (95\% CI 0.51-0.89, p=0.04). The area under the curve for TIMP-1 was 0.78 (95\% CI 0.61-0.94, p=0.007). MMP-9, MMP-2, and TIMP-1 levels did not correlate with gestational age, gender, severity of wheezing.

Conclusion Elevated serum MMP-2 and TIMP-1 levels appear to increase the risk for wheezing. Further studies will be required to determine whether therapeutic inhibitors will prevent recurrent lung morbidities in preterm infants.

\section{SERUM ELECTROLYTES VARIATIONS IN TREATED PATIENTS WITH MODERATE ASTHMA EXACERBATION}

doi:10.1136/archdischild-2012-302724.1712

ML Neamtu, AC Brumar. Pediatric Clinic Hospital, Lucian Blaga University of Sibiu, Sibiu, Romania

Background Salbutamol induces stimulation of beta ${ }_{2}$-receptors resulting in hypokalemia. Corticosteroids also induce plasma electrolytes variations.

Aims

1. To identify blood electrolytes changes following low dose inhaled short-acting beta ${ }_{2}$-agonists;

2. To evaluate if concomitant inhaled corticosteroids treatment can amplify serum electrolytes changes.

Methods We analyzed all children admitted for moderate asthma exacerbation during 6 months period. Inclusion criteria: children between $5-18$ years of age; PEF $>50-75 \%$ of predicted value; serum electrolytes normal ranges. Exclusion criteria: previously treated patients with Salbutamol; Salbutamol hypersensitivity; asthma exacerbation severity levels. The patients were divided into 2 groups: $1^{\text {st }}$ group comprised those treated with beta ${ }_{2}$-agonists and $2^{\text {nd }}$ group is represented by paediatric patients concomitantly treated with beta $_{2}$-agonists and corticosteroids. Both groups were homogenous regarding age and sex ratio. During hospitalization, patients received standard low dose of Salbutamol by metered dose inhaler (MDI) and inhaled Fluticasonum propionate using spacer device with mouthpiece. Included patients were assessed for electrolytes serum levels before treatment and 72 hours after therapy. Data was analyzed statistically using independent sample $\mathrm{T}$ test, skewness, kurtosis.

Results Among 269 admitted patients, 175 children fulfilled inclusion criteria. Both groups structure: 92 children in $1^{\text {st }}$ group, 83 in $2^{\text {nd }}$ group. Authors found for both groups a significant decreasing of serum kalium after beta $_{2}$-agonists treatment ( $p$ value $\left.=0,010\right)$. The study didn't confirm a significant variation of serum electrolytes in $2^{\text {nd }}$ group as compare to $1^{\text {st }}$ group.

Conclusions Study confirmed significant hypokalemia after 72 hours inhaled treatment with beta $_{2}$-agonists; corticosteroids didn't modify kalium level in association with beta ${ }_{2}$-agonists.

\section{REDUCING ASTHMA CLINIC ATTENDANCE USING POSTAL SURVEY WITH MOBILE TEXTING FEEDBACK}

doi:10.1136/archdischild-2012-302724.1713

'LM Perrem, ${ }^{2 P}$ Manning, 'MB 0'Neill. 'Mayo General Hospital, Castlebar; '2Mullingar Regional Hospital, Mullingar, Ireland

Background and Aim Attempting to reduce unnecessary attendances of well patients at outpatient clinics is prudent. This study evaluated the Asthma Control Test (ACT)t and Respiratory Proforma, with feedback through mobile texts, in children with Asthma, to determine attendance at clinic or not.

Methods Patients between 4 and 11 years with a diagnosis of asthma were eligible for inclusion. The parent was surveyed, by ppost, 2 weeks prior to the clinic date and asked to complete the Asthma Control Test (ACT) and a Respiratory Proforma which assessed UACS symptoms, medication usage inclusive of intensification episodes and medical concerns. Mobile telephone numbers were requested. Parents mailed their responses in a supplied stamped envelope supplied. Respondents were divided into 2 categories a) ACT score greater than 19 and a non concerning Respiratory Proforma, who were texted not to attend the clinic but supplied with another outpatient appointment and b) the remainder were texted to attend the clinic.

Results Over 6 clinics the parents of 77 eligible children were surveyed. Fifty eight (75\%) replied of whom 38 (66\%) were well and did not attend the clinic but rebooked. Of 20 who attended, 6 had new symptoms of UACS and 3 had pneumonia. Of 19 who did not reply 7 came to clinic with completed questionnaires, 5 had good control. Ten did not attend the clinic or complete the questionnaire.

Conclusion Asthma care through postal survey with mobile text feedback is an option in the outpatient setting.

\section{ABILITY OF SELF-INFLATING BAGS (SIB) TO DELIVER SUSTAINED INFLATIONS}

doi:10.1136/archdischild-2012-302724.1714

'M Thio, 1,2JA Dawson, ${ }^{3} \mathrm{TJ}$ Moss, ${ }^{3} \mathrm{SB}$ Hooper, ${ }^{1,2} \mathrm{PG}$ Davis. 'Newborn Services, The Royal Women's Hospital; ${ }^{2}$ Murdoch Children's Research Institute; ${ }^{3}$ The Ritchie Centre, MIMR, Monash University, Melbourne, VIC, Australia

Background and Aims In neonatal resuscitation, the use of a sustained inflation (SI) after birth may facilitate lung recruitment. We aimed to assess the ability of several SIB to deliver a SI.

Method In a newborn preterm lamb, we compared 4 different SIB devices fitted with a PEEP valve against a T-piece, using a flow of $8 \mathrm{Lpm}$. Four operators aimed to give 3 targeted SI of $20 \mathrm{cmH}_{2} \mathrm{O}$ (displayed on a manometer) for 30 seconds. The study was repeated with the PEEP valve removed and again with no flow. 
Results 204 combinations were analysed. Mean (SD) duration of SI, maximum inspiratory pressure (maximum IP) and average inspiratory pressure (average IP) are shown in the table.

\section{Abstract 1714 Table 1}

\begin{tabular}{lccc}
\hline Device & duration of SI seconds & maximum IP cmH20 & average IP cmH20 \\
\hline SIB 1 & $6.2(1.6)$ & $30.0(5.8)$ & $17.2(3.3)$ \\
SIB 2 & $13.9(7.9)$ & $24.7(4.7)$ & $17.5(3.5)$ \\
SIB 3 & $4.6(1.2)$ & $23.6(7.7)$ & $12.5(4.7)$ \\
SIB 4 & $33.3(2.3)$ & $26.4(4.6)$ & $20.9(2.5)$ \\
T-piece & $32.6(0.9)$ & $20.0(0)$ & $19.6(0.5)$ \\
\hline
\end{tabular}

PEEP valve removal and absence of flow made no significant difference to the $\mathrm{SI}$ time $(\mathrm{P}=0.34$ and $\mathrm{P}=0.13$ respectively), maximum IP ( $\mathrm{P}=0.17$ and $\mathrm{P}=0.12$ respectively) or average IP $(\mathrm{P}=0.32$ and $\mathrm{P}=0.60$ respectively).

Conclusions SIB perform differently depending on the brand and some are able to deliver sustained SI even in the absence of gas flow. If medically indicated, this may be useful in a resource-limited setting with no gas supply.

\section{TUBERCULOSIS IN CHILDREN - STILL DIAGNOSIS CHALLENGE}

doi:10.1136/archdischild-2012-302724.1715

M Dilberovska, N Uzunovska, D Dacevski. Institute for Respiratory Diseases in ChildrenKozle, Skopje, FYR Macedonia

Aim Retrospective analyze of evaluation for tuberculosis (TB) diagnosis in children.

Materials and Method At the TB Department, for a period of 2007-2011 yr., data of treated patients were evaluated. Analyze was made on the base of diagnostic parameters: anamnesis data (positive TB control, beginning and symptoms of the disease); BCG vaccine and Mantoux test; and results of following investigations: hematology, microbiology, radiology, fiberbronchoscopy, toracocentesis, lumbal punction etc.

Results In the noted period, 267 children with TB were treated at the Department. Primary TB was presented in 229 (85, 8\%) of them. Age group of 5-9 yr. was the most frequent in 147 (55.1\%) children. Positive familiar TB contact was evident in 153 (57.3\%) and positive Mantoux skin test in 179 (67.0\%). Pleural effusion in 21 (7.9\%) and cavernous changes $(4.4 \%)$ were shown on the lung radiograms. In $57(21.3 \%)$ children, lung TB was associated with non-specific disease (pneumonia in 23 (40.4\%). From microbiological investigation: M.tuberculosis (culturally) was positive in 17 (6.6\%); in relation with other bacteria, the most frequent was Haemophilus influenza in $19(9.6 \%)$ children. Fiberbronchoscopy showed changes for TB endobronchitis in $29(10.9 \%)$ children. Other diagnostic procedures were performed in connection with the form of $\mathrm{TB}$.

Conclusion TB diagnostic in children is very difficult to be made. It requires long time period and numbered diagnostic investigations, especially in small children, because of the association with non-specific lung diseases that is very often.

\section{EFFECTS ON GROWTH OF INHALED CORTICOSTEROIDS IN ASTHMATIC CHILDREN}

doi:10.1136/archdischild-2012-302724.1716

'MBI Octavia, 'BM Neamtu, 'NM Leonida, ${ }^{2} \mathrm{M}$ Rodica, ${ }^{3} \mathrm{C}$ Rodica, ${ }^{4} \mathrm{Z}$ Alina, ${ }^{5} \mathrm{M}$ Ionela, ${ }^{6} \mathrm{M}$ Melania, ${ }^{7} \mathrm{~N}$ Cristian, ${ }^{8} \mathrm{~B}$ Radu, ${ }^{4} \mathrm{O}$ Ariela. ${ }^{1}$ Pediatric Hospital, Lucian Blaga University; 2Pneumology, Pediatric Hospital Sibiu; ${ }^{3}$ Pneumology, Pneumopthisiology Hospital Sibiu; ${ }^{4}$ Laboratory Medicine, County Hospital; ${ }^{5}$ Applied Informatics, Faculty of Science, Lucian Blaga University; ${ }^{6}$ Chemistry; ${ }^{7}$ Research; ${ }^{8}$ Pediatric Clinic, Pediatric Hospital Sibiu, Sibiu, Romania
Background Corticosteroids may inhibit growth hormone (GH) axis, reducing $\mathrm{GH}$ release, decreasing tissue expression of growth factors, inhibiting IGF-1 bioactivity, osteoblast activity, promoting bone resorption.

Objectives Evaluating adverse effects of inhaled corticosteroids used in asthmatic children on the following biological parameters: $\mathrm{GH}$ (two measurements), IGF-1(insulin growth factor-1), FAS (alkaline phosphatase), correlated with the presence or absence of atopy (immunoglobulin E levels).

Methods The prospective study included 74 asthmatic children, treated with inhaled corticosteroids aged between 5 and 13 years of age, divided into subgroups. Each type of inhaled glucocorticoid fluticasone, budesonide, mometasone furoate (single or in combination with long-acting bronchodilators) has been analysed for each patient. T-test, Mann-Whitney, Chi-square, binomial tests were used to ascertain the relations between average dose, the duration of treatment and the biological parameters mentioned

Results There were found statistically significant differences $(p<0.05)$ in:

1. patients treated with Seretide $25 / 50$ between the number of patients with $\mathrm{GH}$ values $<1 \mathrm{ng} / \mathrm{ml}$ and number of patients with $\mathrm{GH}>1 \mathrm{ng} / \mathrm{ml}$ (second measurement of $\mathrm{GH}$ ).

2. patients treated with Symbicort $4.5 / 80$, between the number of patients with $\mathrm{GH}$ values $<1 \mathrm{ng} / \mathrm{ml}$ and number of patients with $\mathrm{GH}>1 \mathrm{ng} / \mathrm{ml}$ (first measurement of $\mathrm{GH}$ ).

3. patients treated with Seretide 50/100 for the following parameters: GH (both determinations), IgE and FAS.

Conclusions Systemic effects of fluticasone propionate and budesonide formoterol in small and medium doses were noted in the association with long-acting bronchodilators and were more extensive accordingly to the duration of treatment.

\section{BRONCHOOBSTRUCTIVE SYNDROME IN TUBERCULOSIS IN CHILDHOOD}

doi:10.1136/archdischild-2012-302724.1717

D Dacevski. Institute for Respiratory Diseases in Children-Kozle, Skopje, FYR Macedonia

Bronchoobstructive syndrome in pediatric population, because of its symptomatology, is frequently cause/introduction for detection of etiological moment, and also verification of tuberculosis infection.

Aim To access how often bronchoobstructive crisis are related with respiratory form of tuberculosis (TB) and what forms are the most frequent.

Material and Methods In the period of 12 years (1999-2010) we inspected hospital histories of patients treated because of $\mathrm{TB}$ infection

We noted: anamnesis data for acute respiratory disorder (cough, wheezing, dyspnea...), their long-lasting and expression, clinical finding, laboratory, microbiological and radiological findings, Mantoux test with PPD-5, data about contact with TB ill person, BCGscar etc.

Results In 20,15\% cases with contact known persons these data were neglected and a cause for physician visit was bronchoobstructive episode. Patients from Roman population were the most frequent, and after them Albanians - at the same time social problem was manifested.

Conclusion It is necessary to realize educational and inspecting measures between populations. More attention has to be initiated on the relation physician-parents because of the bigger benefit achieved with early diagnosis and treatment/prevention. 